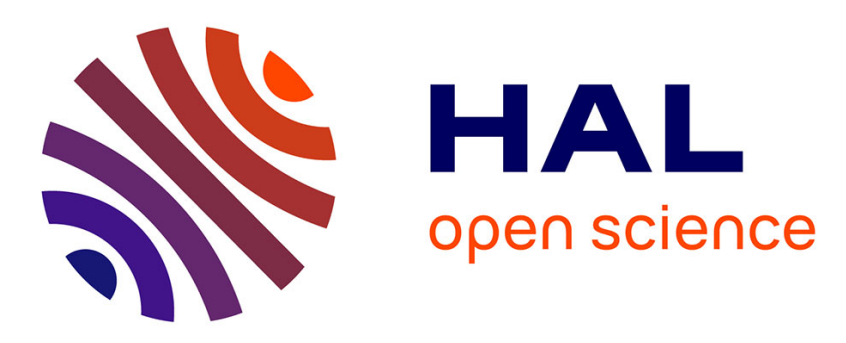

\title{
Trust Evaluation of a System for an Activity with Subjective Logic
}

Nagham Alhadad, Yann Busnel, Patricia Serrano-Alvarado, Philippe Lamarre

\section{To cite this version:}

Nagham Alhadad, Yann Busnel, Patricia Serrano-Alvarado, Philippe Lamarre. Trust Evaluation of a System for an Activity with Subjective Logic. TrustBus, Sep 2014, Munich, Germany. pp.48-59. hal-01059198

\section{HAL Id: hal-01059198 https://hal.science/hal-01059198}

Submitted on 29 Aug 2014

HAL is a multi-disciplinary open access archive for the deposit and dissemination of scientific research documents, whether they are published or not. The documents may come from teaching and research institutions in France or abroad, or from public or private research centers.
L'archive ouverte pluridisciplinaire HAL, est destinée au dépôt et à la diffusion de documents scientifiques de niveau recherche, publiés ou non, émanant des établissements d'enseignement et de recherche français ou étrangers, des laboratoires publics ou privés. 


\title{
Trust Evaluation of a System for an Activity with Subjective Logic
}

\author{
Nagham Alhadad ${ }^{1,2}$, Yann Busnel ${ }^{1}$, Patricia Serrano-Alvarado ${ }^{1}$, Philippe Lamarre $^{3}$ \\ ${ }^{1}$ LINA/Université de Nantes - France \\ ${ }^{2}$ LIG/Université de Grenoble Alpes - France \\ ${ }^{3}$ LIRIS/INSA Lyon - France
}

\begin{abstract}
Recently, trust emerged as a momentous aspect to evaluate resources, services or persons. In our work, the trust notion focuses on a system as a whole and from the point of view of a particular user to do a particular digital activity as editing a document, mailing, chatting, etc. Our general goals are (i) to enable users to have a personal comparison of applications allowing them to do an activity such that they can choose the one satisfying their personal expectations and (ii) to know how trustworthy their system is to do a particular activity (all applications together). We consider a system as a graph composed of paths where the source is a person and the target is a final application or data. We consider that trust in a system depends on its architecture and we identify two problems (i) how to evaluate trust in a graph having dependent paths i.e., paths having common nodes, and (ii) how to express and deal with uncertainty in evaluating trust in a system. Concerning the first problem, trust approaches based on graphs have been proposed in the domain of social networks. Their solution for dependent paths is either removing paths or just choosing one of them what causes loss of information. Considering the second problem, subjective logic emerged to express trust as a subjective opinion with a degree of uncertainty. In this paper we present SUBJECTIVETRUST, an approach that relies on subjective logic to evaluate trust in distributed systems. It proposes two solutions to treat dependent paths and takes into account the shape of the system architecture in trust evaluation. We analyze SUBJECTIVETRUST in a series of experiments that show its accuracy.
\end{abstract}

\section{Introduction}

When users need to choose a system to perform a digital activity, like editing a document or mailing, they face several available options. To choose a system, they evaluate many criteria as functionality, ease of use, QoS, or economical aspects. Trust also emerged as a momentous aspect of choice [13]. Evaluating trust in a system is complex and becomes more challenging when systems use distributed architectures. Our general goals are $(i)$ to enable users to have a personal comparison of applications allowing them to do an activity such that they can choose the one satisfying their personal expectations and (ii) to know how trustworthy their system is to do a particular activity (all applications together). We argue that studying trust in the separate entities that compose a system does not give a picture of how trustworthy a system is as a whole. Indeed, the trust in a system depends on its entities but also on its architecture. More precisely, on the way the entities, the users depends on to do their activities, are organized. 
Trust has been studied from different points of views $[5,6,17]$ and to evaluate it metrics vary from binary, scalar to probabilistic approaches [13,18]. As users hardly have all information to provide a dogmatic opinion on something or someone, subjective logic [10], an extension of classical probability, emerged to express trust as a subjective opinion with a degree of uncertainty.

We consider a system as a graph [3] composed of paths where the source is a person and the target a final application or data. Intermediary nodes are entities (software) allowing to achieve the activity. Each path is a way to do a given activity. Trust approaches based on graphs $[7,9,11,12,14,16]$ are especially used in the context of social networks where the main idea to derive trust is to propagate it through a path then through a social graph [1]. Their solution for dependent paths is either removing paths or just choosing one of them in such a way the obtained graph has only independent paths what causes loss of information.

In a former work, we proposed SocIOTRUST, an approach to evaluate trust based on probability theory [4]. In this paper, we aim to take advantage of the benefits of subjective logic and we present SUBJECTIVETRUST, an approach to evaluate trust in distributed system architectures that relies on subjective logic. The goal is to allow a person to evaluate her trust in a system for an activity from her potentially uncertain trust in each node of the system graph. Although our approach relies on a graph, like in the social network domain, the interpretation of the graph is different. For us, a graph represents a system for a digital activity and not a social network. This assumption plays an important role in the operations we apply and in the results we interpret. SUBJECTIVETRUST estimates trust at two levels of granularities, namely, trust in a path and trust in a system. We address the problem of dependent paths in a graph and we propose two solutions. We evaluate SUBJECTIVETRUST in a series of experiments that compare the proposed solutions and analyze their accuracy.

This paper is organized as follows. Section 2 gives a quick overview of subjective logic and presents related works. Section 3 introduces SUBJECTIVETRUST. We present the experiments that validate our approach in Section 4 before concluding in Section 5.

\section{Background and related works}

Subjective logic has been proposed recently as a formalism to express uncertainty [10]. In this paper we do not propose enhancements to this logic, we just adopt it to the context of personal evaluation of trust in a system for an activity. Next section gives an overview of subjective logic ( $c f$. Section 2.1). We then explicit the problem of dependent paths in graph-based trust approaches and present related works ( $c f$. Section 2.2).

\subsection{Overview of subjective logic}

Several metrics have been proposed to evaluate trust. In binary metrics, trust values are only trust or distrust [8]. In simple metrics, trust values are scaled intervals formed from relatively simple methods of computation like a multiplication or a weighted average [7]. In probabilistic metrics, a trust value represents the probability of how much likely a trustor will perform actions as the trustee expects. In these metrics, a given 
person cannot express her ignorance or her degree of uncertainty about a proposition because there is no value that means "I do not know" or "I am not sure". This idea led researchers to look for mathematical formalisms to express uncertainty.

Subjective logic [10], an extension of classical probability, proposes a solution to this problem. It is a probabilistic logic that uses opinions as input and output variables. Opinions explicitly express uncertainty about probability values, and can express degrees of ignorance about a proposition. In the terminology of subjective logic, an opinion held by an individual $P$ about a proposition $x$ is the ordered quadruple $O_{x}=\left(b_{x}, d_{x}, u_{x}, a_{x}\right)$, where $b_{x}$ (belief) is the belief that $x$ is true, $d_{x}$ (disbelief) is the belief that $x$ is false, and $u_{x}$ (uncertainty) is the amount of uncommitted belief, $b_{x}, d_{x}, u_{x} \in[0 . .1]$ and $b_{x}+d_{x}+u_{x}=1$. The last value $a_{x} \in[0 . .1]$ is called the base rate. In the absence of any specific evidence about a given party, the base rate determines the default trust. An opinion's probability expectation value, which can be determined as $E\left(O_{x}\right)=b_{x}+a_{x} u_{x}$, is interpreted as a probability measure indicating how $x$ is expected to behave in the future. More precisely, $a_{x}$ determines how uncertainty shall contribute to the probability expectation value $E\left(O_{x}\right)$. Subjective logic consists of a set of logical operations which are defined to combine opinions.

- Conjunction operator $(\wedge)$ represents the opinion of a person on several propositions.

- Disjunction operator $(\vee)$ represents the opinion of a person on one of the propositions or any union of them.

- Discounting operator $(\otimes)$ represents the transitivity of the opinions.

- Consensus operator $(\oplus)$ represents the consensus of opinions of different persons.

In this work, we use subjective logic to evaluate trust.

\subsection{Graph-based trust approach}

Trust approaches based on graphs $[1,8,11,12,15,16]$ are especially used in social networks where the main idea of trust derivation is to propagate it between two nodes in a graph that represents the social network. A social network is a social structure composed of a set of persons (individuals or organizations) and a set of relations among these persons. It can be represented as a graph where the nodes are the persons and the edges are the relations between them. Trust between two persons in a social network can be evaluated based on this graph where the source node is the trustor, the target node is the trustee and the other nodes are the intermediate nodes between the trustor and the trustee. Values are associated with the edges to represent the trust value attributed by the edge source node towards the edge target node. Figure 1 shows an example of trust relationships in a social network. For instance, $B$ trusts $C$ with the value 0.8 .

Trust propagation focuses on finding a trust value from a person towards another given person through the multiple paths that relate them. For instance, in Figure 1, how much $A$ trusts $E$ knowing that there are two paths that relate $A$ with $E$ ? The paths are: path $_{1}=\{A, B, C, E\}$, and $p a t h_{2}=\{A, B, D, E\}$. In [1], authors propose a general approach for graph-based trust. They divide the process of trust evaluation into two steps: 


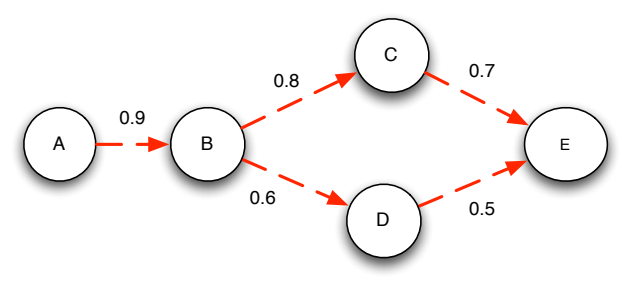

\begin{tabular}{|c|c|}
\hline$\otimes$ average & $\otimes$ multiplication \\
$\oplus$ maximum & $\oplus$ comultiplication \\
\hline Relation 1: & Relation 1: \\
$T_{E}^{A}=0.825$ & $T_{E}^{A}=0.623$ \\
\hline Relation 2: & Relation 2: \\
$T_{E}^{A}=0.8$ & $T_{E}^{A}=0.64$ \\
\hline
\end{tabular}

Fig. 1: The different obtained results of Relations 1, 2 by applying an example of discrete metrics and continuous metrics on a simple graph

1. Trust combination through a path: the main idea is to combine the trust values among the intermediate edges of a path to obtain a trust value though this path. Several operators are employed ranging from basic operators like the minimum to new operators like the discounting operator of subjective logic.

2. Trust combination through a graph: the main idea is to combine the several trust values through the multiple paths, which relate the source with the target, to obtain a single trust value through the whole graph. Several operators are employed to combine trust through a graph, ranging from basic operators like the average to new ones like the consensus operator of subjective logic.

In [11,12], Jøsang et al. raised a problem of graph-based trust approaches if trust is evaluated through the previous two steps. They argue that some metrics do not give exact results when there are dependent paths i.e., paths that have common edges in the graph. To explain this problem, we give a simple example shown in Figure 1. We need to evaluate $T_{E}^{A}$ corresponding to A's trust value in $E$. The paths between $A$ and $E$ are: path $_{1}=\{A, B, C, E\}$ and path $_{2}=\{A, B, D, E\}$. There is a common edge between these two paths which is $A \longrightarrow B$. Let $\otimes$ be the operator of trust combination through a path and $\oplus$ be the operator of trust combination through a graph. To evaluate $T_{E}^{A}$, the A's trust value in $E$ :

$$
T_{E}^{A}=T_{B}^{A} \otimes\left(\left(T_{C}^{B} \otimes T_{E}^{C}\right) \oplus\left(T_{D}^{B} \otimes T_{E}^{D}\right)\right)
$$

However, if we apply the previous two steps, $T_{E}^{A}$ is computed as follows:

$$
T_{E}^{A}=\left(T_{B}^{A} \otimes T_{C}^{B} \otimes T_{E}^{C}\right) \oplus\left(T_{B}^{A} \otimes T_{D}^{B} \otimes T_{E}^{D}\right)
$$

Relations 1, 2 consist of the same two paths path $h_{1}$ and $p^{2} h_{2}$, but their combined structures are different. $T_{B}^{A}$ appears twice in Relation 2. In some metrics, the previous two equations produce different results. For instance, when implementing $\otimes$ as binary logic "AND", and $\oplus$ as binary logic "OR", the results would be equal. However, when implementing $\otimes$ and $\oplus$ as probabilistic multiplication and comultiplication respectively, the results would be different. If $\otimes$ is the minimum function and $\oplus$ is the average function, the results are also different. Figure 1 shows the application of different operators on the example of our simple graph and the different obtained results of Relations 1 and 2 .

In graph-based trust approaches, this problem is either ignored [16], either simple solutions are proposed like choosing one path in a graph [15], or removing the paths 
that are considered unreliable [8,12]. In [12], Jøsang et al. propose a method based on graph simplification and trust derivation with subjective logic named, Trust Network Analysis with Subjective Logic (TNA-SL). They simplify a complex trust graph into a graph having independent paths by removing the dependent paths that have a high value of uncertainty. The problem of the previous solution is that removing paths from a graph could cause loss of information. To solve this problem, in [11], authors propose to transform a graph that has dependent paths into a graph that has independent paths by duplicating the edges in common and splitting the associated opinions to them.

In SOCIOTRUST [4], a graph-based trust approach based on probability theory to evaluate trust in a system for an activity, the problem of dependent paths is solved using conditional probability. In SOCIOTRUST, trust values are considered as the probability by which a trustor believes that a trustee behaves as expected [13]. SOcioTrust is an approach that works perfectly in full-knowledge environments. However, in uncertain environments, users might not be in possession of all the information to provide a dogmatic opinion and traditional probability cannot express uncertainty.

NA: In this paper, we rely on a graph to evaluate trust like in the social network domain, but the interpretation of the graph is different. For us, a graph represents a system for a digital activity and not $a$ social network. This assumption plays an important role in the operations we apply for trust evaluation. For instance, in social network, to evaluate trust through a path using subjective logic, the operator of discounting $(\otimes)$ is used to compute the transitivity through a path, whereas, in our work evaluating trust in a path is the trust in the collection of the nodes that form this path. In the same manner, to evaluate trust through a graph in social network, the operator of consensus $(\oplus)$ is used to evaluate the consensus of opinions of different persons through the different paths that form the graph, whereas, in our work, paths represent the ways a user follows to achieve an activity so evaluating trust in a graph is the trust in one of the paths or any union of them.

Next Section presents SubjectiveTrust, the contribution of this paper that is based on subjective logic to deal with uncertainty. It faces the problem of dependent paths by proposing two methods, Copy and Split. We provide these methods with the necessary formalisms and algorithms to be applied to the context of our work.

\section{SubJeCtiveTrust}

In this approach, the graph represents an architecture allowing an activity to be achieved. The source node in a graph is the user who performs an activity and the target node is a data instance or an application that is related to this activity [3]. Each path between the source node and the target node represents a way to achieve the activity through a system. User's opinions are associated with the nodes and not the edges as in social networks because they represent the local user's opinions on these nodes. Whereas in social networks the associated values to the edges represent the trust between the nodes related by the edges ${ }^{4}$.

\footnotetext{
${ }^{4}$ For more details about obtaining a graph of a system allowing an activity to be achieved, see our previous work SOCIOTRUST [4].
} 
We aim to evaluate trust towards a whole graph that represents an activity achieved through a system. To do that, we pass through two steps opinion on a path (cf. Section 3.1) and opinion on a system (cf. Section 3.2), both for an activity achieved by a user. In our graph, dependent paths are the ones that have common nodes and not common edges because opinions are associated with nodes in our approach. To solve the problem of dependent paths, we propose two methods named, Copy and Split with their necessary formalisms and algorithms to be applied to the context of our work. In both, we consider duplicating the common nodes in order to obtain two independent opinions associated with them. In Copy, we also duplicate the opinions associated with the common nodes. Split is inspired from [11], after duplicating the common nodes, the associated opinions to them are also split. In the following sections, we denote a path by $\sigma$ and a system by $\alpha$. A path in our graph does not consider the source and the target node.

\subsection{Opinion on a path for an activity}

When a user needs to achieve an activity through a path, she needs to pass through all the nodes composing this path. Hence, an opinion on a path is a composition of the opinions on all the nodes composing this path.

The conjunction operator in subjective logic represents the opinion of a person on several propositions. If $O_{x}^{P}=\left(b_{x}^{P}, d_{x}^{P}, u_{x}^{P}, a_{x}^{P}\right)$ is $P$ 's opinion on $x$ and $O_{y}^{P}=$ $\left(b_{y}^{P}, d_{y}^{P}, u_{y}^{P}, a_{y}^{P}\right)$ is $P$ 's opinion on $y, O_{x \wedge y}^{P}$ represents $P$ 's opinion on both $x$ and $y$. Thus, the conjunction operator is the appropriate operator to compute an opinion on a path from the opinions on the nodes.

Let $\sigma=\left\{N_{1}, N_{2}, \ldots, N_{n}\right\}$ be a path that enables a user $P$ to achieve an activity. $P$ 's opinion on the nodes $\left\{N_{i}\right\}_{i \in[1 . . n]}$ for an activity are denoted by $O_{N_{i}}=$ $\left(b_{N_{i}}, d_{N_{i}}, u_{N_{i}}, a_{N_{i}}\right)$. P's opinion on the path $\sigma$ for achieving an activity, denoted by $O_{\sigma}=\left(b_{\sigma}, d_{\sigma}, u_{\sigma}, a_{\sigma}\right)$ can be derived by the conjunction of $P$ 's opinions on $\left\{N_{i}\right\}_{i \in[1 . . n]} . O_{\sigma=\left\{N_{1}, \ldots, N_{n}\right\}}=\bigwedge\left\{O_{N_{i}}\right\}_{i \in[1 . . n]}$. Given the following relations from [10], we have:

$$
O_{x \wedge y}=\left\{\begin{array}{l}
b_{x \wedge y}=b_{x} b_{y} \\
d_{x \wedge y}=d_{x}+d_{y}-d_{x} d_{y} \\
u_{x \wedge y}=b_{x} u_{y}+u_{x} b_{y}+u_{x} u_{y} \\
a_{x \wedge y}=\frac{b_{x} u_{y} a_{y}+b_{y} u_{x} a_{x}+u_{x} a_{x} u_{y} a_{y}}{b_{x} u_{y}+u_{x} b_{y}+u_{x} u_{y}}
\end{array}\right.
$$

We obtain the following generalization for the opinion on a path $\sigma$ :

$$
O_{\sigma=\left\{N_{1}, \ldots, N_{n}\right\}}=\left\{\begin{array}{l}
b_{\sigma=\left\{N_{1}, \ldots, N_{n}\right\}}=b_{\bigwedge\left\{N_{i}\right\}_{i \in[1 . . n]}}=\prod_{i=1}^{n} b_{N_{i}} \\
d_{\sigma=\left\{N_{1}, \ldots, N_{n}\right\}}=d_{\bigwedge\left\{N_{i}\right\}_{i \in[1 . . n]}=1-\prod_{i=1}^{n}\left(1-d_{N_{i}}\right)} \\
u_{\sigma=\left\{N_{1}, \ldots, N_{n}\right\}}=u_{\bigwedge\left\{N_{i}\right\}_{i \in[1 . . n]}}=\prod_{i=1}^{n}\left(b_{N_{i}}+u_{N_{i}}\right)-\prod_{i=1}^{n}\left(b_{N_{i}}\right) \\
a_{\sigma=\left\{N_{1}, \ldots, N_{n}\right\}}=a_{\bigwedge\left\{N_{i}\right\}_{i \in[1 . . n]}}=\frac{\prod_{i=1}^{n}\left(b_{N_{i}}+u_{N_{i}} a_{N_{i}}\right)-\prod_{i=1}^{n}\left(b_{N_{i}}\right)}{\prod_{i=1}^{n}\left(b_{N_{i}}+u_{N_{i}}\right)-\prod_{i=1}^{n}\left(b_{N_{i}}\right)}
\end{array}\right.
$$

Due to space constrains, proofs of Relation 4 and the verifications of the correction (i.e., $b_{\sigma}+d_{\sigma}+u_{\sigma}=1,0<b_{\sigma}, d_{\sigma}, u_{\sigma}, a_{\sigma}<1$ ) are not presented here. The interested reader is invited to read the companion paper to the present work where all our proofs are developed [2]. 


\subsection{Opinion on a system for an activity}

A system, which often contains several paths, represents the several ways a user can achieve her activity. After building opinions on all paths, an opinion on a system can be built. An opinion on a system is the opinion of a person on one of the paths or any union of them.

The disjunction operator in subjective logic represents the opinion of a person on one or several propositions. If $O_{x}^{P}=\left(b_{x}^{P}, d_{x}^{P}, u_{x}^{P}, a_{x}^{P}\right)$ is $P$ 's opinion on $x$ and $O_{y}^{P}=$ $\left(b_{y}^{P}, d_{y}^{P}, u_{y}^{P}, a_{y}^{P}\right)$ is $P$ 's opinion on $y, O_{x \vee y}^{P}$ represents $P$ 's opinion on $x$ or $y$ or both. Thus, the disjunction operator is the appropriate operator to evaluate an opinion on a system. In the following, we show how to build an opinion on a system when $(i)$ there are not common nodes among paths and (ii) there are common nodes among paths.

Opinion on a system having independent paths: let $\left\{\sigma_{1}, \sigma_{2}, \ldots, \sigma_{m}\right\}$ be the paths that enable a user $P$ to achieve an activity. The user opinion on the paths $\left\{\sigma_{i}\right\}_{i \in[1 . . m]}$ for an activity are denoted by $O_{\sigma_{i}}=\left(b_{\sigma_{i}}, d_{\sigma_{i}}, u_{\sigma_{i}}, a_{\sigma_{i}}\right)$. The user opinion on the system $\alpha$ for achieving the activity, denoted by $O_{\alpha}=\left(b_{\alpha}, d_{\alpha}, u_{\alpha}, a_{\alpha}\right)$ can be derived by the disjunction of $P$ 's opinions on $\left\{\sigma_{i}\right\}_{i \in[1 . . m]} . O_{\alpha}=\bigvee\left\{O_{\sigma_{i}}\right\}_{i \in[1 . . m]}$. Given the following relations from [10]:

$$
O_{x \vee y}=\left\{\begin{array}{l}
b_{x \vee y}=b_{x}+b_{y}-b_{x} b_{y} \\
d_{x \vee y}=d_{x} d_{y} \\
u_{x \vee y}=d_{x} u_{y}+u_{x} d_{y}+u_{x} u_{y} \\
a_{x \vee y}=\frac{u_{x} a_{x}+u_{y} a_{y}-b_{x} u_{y} a_{y}-b_{y} u_{x} a_{x}-u_{x} a_{x} u_{y} a_{y}}{u_{x}+u_{y}-b_{x} u_{y}-b_{y} u_{x}-u_{x} u_{y}}
\end{array}\right.
$$

We obtain the following generalization for the opinion on a system $\alpha:$ :

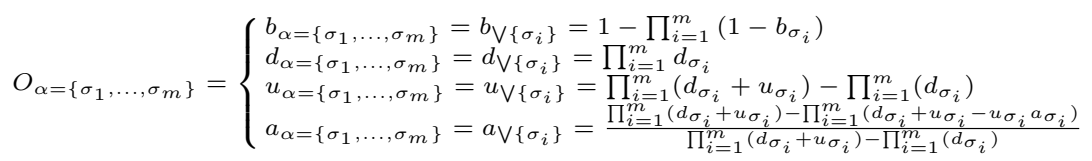

The proofs of Relation 6 and the verifications of the relations: $b_{\alpha}+d_{\alpha}+u_{\alpha}=1$, $0<b_{\alpha}<1,0<d_{\alpha}<1,0<u_{\alpha}<1$ and $0<a_{\alpha}<1$ are developed in [2].

Opinion on a system having dependent paths: in subjective logic as in probabilistic logic, the disjunction is not distributive over the conjunction, i.e., we have $O_{x} \wedge\left(O_{y} \vee\right.$ $\left.O_{z}\right) \neq\left(O_{x} \wedge O_{y}\right) \vee\left(O_{x} \wedge O_{z}\right)$. This is due to the fact that opinions must be assumed to be independent, whereas distribution always introduces an element of dependence. In SocioTRust [4], this problem has been resolved by using conditional probability. Then when there are common nodes among paths, Relations 4 and 6 cannot be applied directly. In order to apply subjective logic for evaluating trust in a system, we propose to transform a graph having dependent paths to a graph having independent paths. Once this transformation is made, we can apply the Relations 4 and 6 . To do that, two methods are proposed Copy and Split. 


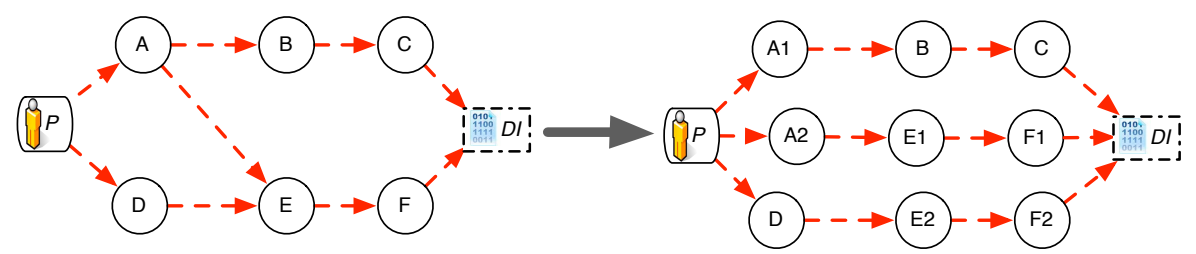

Fig. 2: Graph transformation using node splitting.

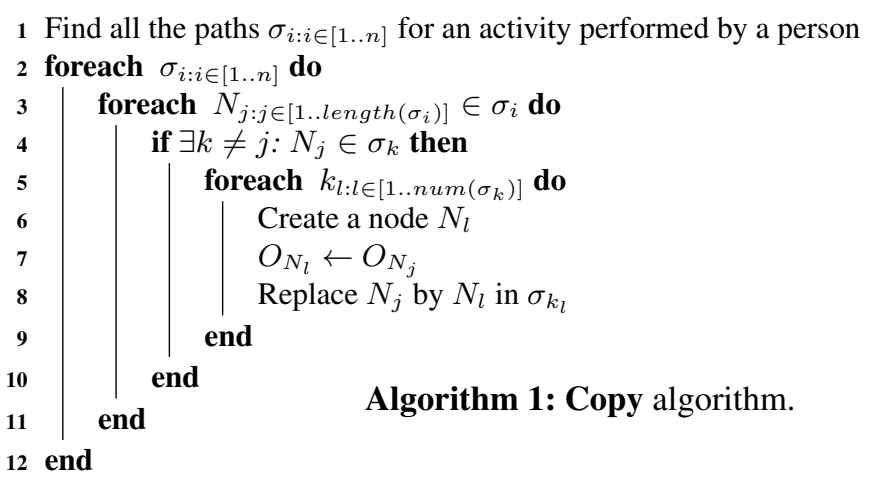

Copy: this method is achieved by duplicating a common node into several different nodes as illustrated in Figure 2. The left side of this figure shows an example of a graph that has three dependent paths. The source node is $P$ and the target node is $D I$. The dependent paths are: $\sigma_{1}=\{A, B, C\}, \sigma_{2}=\{A, E, F\}$ and $\sigma_{3}=\{D, E, F\}$. The common nodes are $A, E$ and $F$. For instance, $A$ is a common node between $\sigma_{1}$ and $\sigma_{2}$. By applying Copy, $A$ becomes $A_{1}, A_{2}$ such that in the new graph, $A_{1} \in \sigma_{1}^{\prime}=\{A 1, B, C\}$ and $A_{2} \in \sigma_{2}^{\prime}=\{A 2, E, F\}$, so is the case for the nodes $E$ and $F$. The right part of Figure 2 shows the new graph after duplicating the common nodes. The new graph contains the paths $\sigma_{1}^{\prime}=\{A 1, B, C\}, \sigma_{2}^{\prime}=\{A 2, E 1, F 1\}$ and $\sigma_{3}^{\prime}=\{D, E 2, F 2\}$. Concerning opinions, we keep the same opinion associated with the original node on the duplicated nodes. This method is based on the idea that the new produced path $\sigma^{\prime}$ maintains the same opinion of the original path $\sigma$. In this case $O_{\sigma_{1}}=O_{\sigma_{1}^{\prime}}$ and $O_{\sigma_{2}}=O_{\sigma_{2}^{\prime}}$. This method is shown in Algorithm 1.

Split: similar to Copy, nodes are duplicated to obtain independent paths as shown in Figure 2. In order to maintain the opinion on the global system, we split the opinion on the dependent node into independent opinions, such that their disjunction produces the original opinion. Formally speaking, if node $A$ is in common between $\sigma_{1}$ and $\sigma_{2}$ and the opinion on $A$ is $O_{A}, A$ is duplicated into $A_{1} \in \sigma_{1}^{\prime}$ and $A_{2} \in \sigma_{2}^{\prime}$ and the opinion $O_{A}$ is split into $O_{A_{1}}$ and $O_{A_{2}}$ where $O_{A_{1}}$ and $O_{A_{2}}$ satisfy the following relations: $O_{A_{1}}=O_{A_{2}}$ and $O_{A_{1}} \vee O_{A_{2}}=O_{A}$. 


$$
\bigwedge\left\{\begin{array} { l } 
{ O _ { A _ { 1 } } \vee \ldots \vee O _ { A _ { n } } = O _ { A } } \\
{ O _ { A _ { 1 } } = \ldots = O _ { A _ { n } } }
\end{array} \Rightarrow \left\{\begin{array}{l}
b_{A 1}=b_{A 2}=\ldots=b_{A_{n}}=1-\left(1-b_{A}\right)^{\frac{1}{n}} \\
d_{A 1}=d_{A 2}=\ldots=d_{A_{n}}=d_{A}^{\frac{1}{n}} \\
u_{A 1}=u_{A 2}=\ldots=u_{A_{n}}=\left(d_{A}+u_{A}\right)^{\frac{1}{n}}-d_{A}^{\frac{1}{n}} \\
a_{A 1}=a_{A 2}=\ldots=a_{A_{n}}=\frac{\left(1-b_{A}\right)^{\frac{1}{n}}-\left(1-b_{A}-a_{A} u_{A}\right)^{\frac{1}{n}}}{\left(d_{A}+u_{A}\right)^{\frac{1}{n}}-d_{A} \frac{1}{n}}
\end{array}\right.\right.
$$

The proofs of Relation 7 are developed in [2]. Split algorithm is made by replacing Line 7 in Copy Algorithm by: " $O_{N_{j k}} \leftarrow$ opinion resulted from Relation 7".

\section{Experimental evaluation}

In this section, we compare Copy and Split to a modified version of TNA-SL [12], that is based on simplifying the graph by deleting the dependent paths that have high value of uncertainty ( $c f$. Section 2.2). In TNA-SL, after the graph simplification, trust is propagated. In our work, trust is not propagated and a comparison to a propagation approach has no sense. Thus, we modify TNA-SL such that trust evaluation is made by applying Relations 4 and 6 introduced in Section 3. We call this method a modified TNA-SL (mTNA).

The objectives of the experiments are (i) to compare Copy and Split to mTNA to verify their behavior and observe the differences among the results, and (ii) to evaluate their accuracy. Next sections present the experiments, their results, analysis and interpretation.

\subsection{Comparing the proposed methods}

To tackle the first objective, we experiment with a graph that contains only independent paths. The three methods, mTNA, Copy and Split give the same exact results as expected because the three of them follow the same computational model when graphs contain only independent paths. Then, we experiment on a graph that has relatively high rate of common nodes and dependent paths. $75 \%$ of the paths of the chosen graph are dependent paths and $60 \%$ of nodes are common nodes.

In our experiments, random opinions $O_{N}=\left(b_{N}, d_{N}, u_{N}, a_{N}\right)$ are associated with each node, and the opinion's probability expectation value of the graph, $\mathbb{E}\left(O_{\alpha}\right)=b_{\alpha}+$ $a_{\alpha} u_{\alpha}$ is computed using the three methods, mTNA, Copy and Split. This experiment is repeated 50 times where each time represents random opinions of a person associated with the different nodes that compose the graph. We analyze the opinion's probability expectation values of the graph, $\mathbb{E}\left(O_{\alpha}\right)=b_{\alpha}+a_{\alpha} u_{\alpha}$ and not all the opinion parameters $O_{\alpha}=\left(b_{\alpha}, d_{\alpha}, u_{\alpha}, a_{\alpha}\right)$.

Figure 3 shows obtained results. We notice that the three methods almost have the same behavior, when the $\mathbb{E}\left(O_{\alpha}\right)$ increases in one method it increases in the other methods, and vice versa. We also observe some differences among the three methods that are not always negligible like at experience 9 and 40 in Figure 3. This observation led us to the question: which of these methods give the most accurate results? To evaluate the accuracy of Split, Copy and mTNA, we conduct other experiments explained in the next section. 


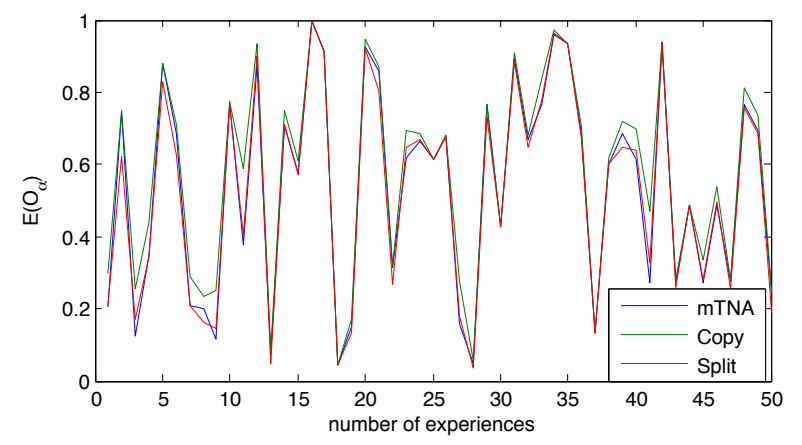

Fig. 3: Value of $\mathbb{E}\left(O_{\alpha}\right)$ for 50 persons using the three methods mTNA, Copy and Split.

\subsection{Studying the accuracy of the proposed methods}

SOCIOTRUST [4], that uses theory of probability to evaluate trust in a system, has the advantages that it has no approximations in case there are dependent paths thanks to conditional probability ( $c f$. Section 2.2). Thus it works perfectly if users are sure of their judgments of trust i.e., the values of uncertainty are equal to 0 .

Subjective logic is equivalent to traditional probabilistic logic when $b+d=1$ such that $u=0$, i.e., the value of uncertainty is equal to 0 . When $u=0$, the operations in subjective logic are directly compatible with the operations of the traditional probability. In this case the value of $\mathbb{E}(O)=b+a u=b$ corresponds to the value of probability.

Since SOCIOTRUST is based on probability theory, the obtained results by applying subjective logic if $u=0$ should be equal to the ones using probability theory. We can evaluate the accuracy of the proposed methods by setting $u=0$ and comparing the value of $b_{\alpha}=\mathbb{E}\left(O_{\alpha}\right)$ resulted from applying the three methods to the trust value obtained by applying SOCIOTRUST.

The experiments are conducted on the graph of Section 4.1. Random opinions $O_{N}=\left(b_{N}, d_{N}, 0, a_{N}\right)$ are associated with each node, and the probability expectation of the graph $\mathbb{E}\left(O_{\alpha}\right)=b_{\alpha}+a_{\alpha} u_{\alpha}=b_{\alpha}$ is computed.

For simplicity, the notations $T_{S T}, T_{\mathrm{MTNA}}, T_{\mathrm{COPY}}, T_{\mathrm{SPLIT}}$ respectively denote system's trust value resulting from applying SOCIOTRUST and system's opinion probability expectation resulting from applying mTNA, Copy and Split.

To make our comparision of $T_{S T}$ versus $T_{\mathrm{MTNA}}, T_{\mathrm{COPY}}, T_{\mathrm{SPLIT}}$, we simply compute the subtractions between them i.e., $T_{S T}-T_{\mathrm{MTNA}}, T_{S T}-T_{\mathrm{COPY}}, T_{S T}-T_{\mathrm{SPLIT}}$. The average of each of the previous values are computed through 10000 time to give a reliable average. The standard deviation $(S D)$ is also computed to show how much variation from the average exists in the three cases. Figure 4 shows obtained results.

As we notice from Figure 4, Copy is the method that gives the closest results to SOCIOTRUST, the average of the difference of its result when $u=0$ and the result of traditional probability over 10000 times is equal to 0.014 , which is an indication that this method gives the nearest result to the exact result and its average error rate is around $1.4 \%$.

The average error rate of mTNA (2.4\%) is less than Split (3.2\%), but the standard deviation of mTNA is 0.045 where in Split, it is 0.037 . That means that in some cases, 


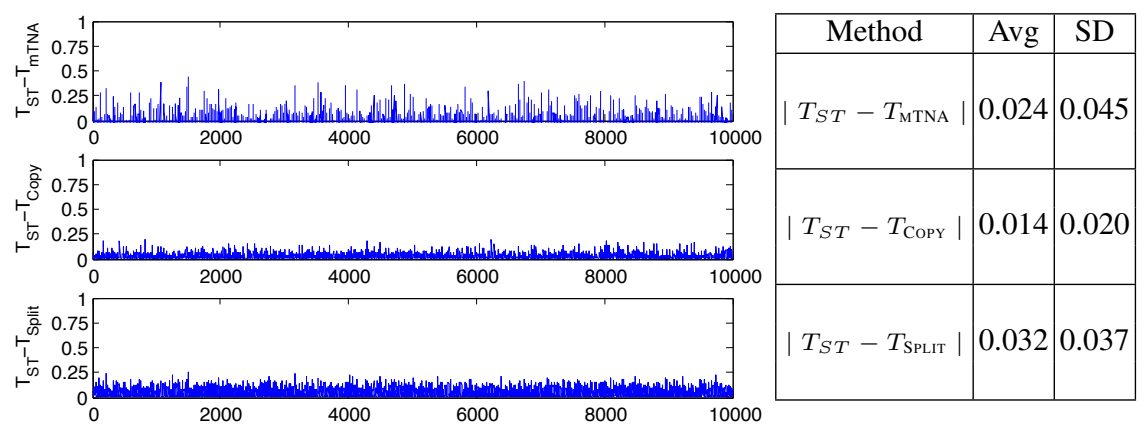

Fig. 4: The difference between the opinion's probability expectation of a graph $\mathbb{E}\left(O_{\alpha}\right)$ using mTNA, Copy and Split when $u=0$ and the trust value resulting from using SOCIOTRUST.

mTNA can give results that are farther than Split from the exact results. Thus, Split shows a more stable behavior than mTNA.

Copy shows the most convincing result. The average error rate is around 0.014 and the standard deviation is 0.02 .

The objective of this experiment is not criticizing the proposed methods in the literature for the problem of dependent paths. These methods are proposed to deal with the problem of trust propagation through a graph, whereas, in our work we focus on evaluating trust towards the whole graph. The employed operators in our case are different from the employed operators in trust propagation. TNA-SL or any proposed method in the literature can work properly in their context.

In this experiment, we show that Copy, our new proposed method, is the method the more adaptable to be used with respect to the context of our work. Extensive simulations on different types of graphs are provided in [2] and follow the same behavior presented above.

\section{Conclusion and perspectives}

This paper presents SUBJECTIVETRUST, a graph-based trust model to evaluate user's trust in a system for an activity from their trust in nodes in the system graph. SUBJECTIVETRUST uses subjective logic to allow users to express their uncertainties in their jugement of trust. We propose two methods to face the problem of dependent paths in a graph for evaluating trust and through our experiments we show their accuracy in our contexte.

Our previous work [4], named SOcIOTRUST, that uses traditional probability, was confronted to real users through a real case-study. In SOCIOTRUST, 25\% of users were not satisfied of the obtained results because they were not able to express their uncertainties about trust values using the traditional probability. SUBJECTIVETRUST allows users to express their uncertainty because it is based on subjective logic. In a future work, we aim to confront SUBJECTIVETRUST approach to real users through a real case-study. 


\section{References}

1. I. Agudo, C. Fernandez-Gago, and J. Lopez. A Model for Trust Metrics Analysis. In Proceedings of the 5th international conference on Trust, Privacy and Security in Digital Business (TrustBus), pages 28-37, 2008.

2. N. Alhadad, Y. Busnel, P. Serrano-Alvarado, and P. Lamarre. Graph-Based Trust Model for Evaluating Trust Using Subjective Logic. Technical Report hal-00871138, LINA - CNRS : UMR6241, Oct. 2013.

3. N. Alhadad, P. Lamarre, Y. Busnel, P. Serrano-Alvarado, M. Biazzini, and C. Sibertin-Blanc. SocioPath: Bridging the Gap between Digital and Social Worlds. In Proceedings of the 23rd International Conference on Database and Expert Systems Applications (DEXA), pages 497 505, 2012.

4. N. Alhadad, P. Serrano-Alvarado, Y. Busnel, and P. Lamarre. Trust Evaluation of a System for an Activity. In Proceedings of the 10th International Conference on Trust, Privacy \& Security in Digital Business, (TrustBus), pages 24-36, 2013.

5. K. Cook. Trust in Society. New York: Russell Sage Foundation, 2001.

6. D. Gambetta. Can we Trust Trust. Trust: Making and breaking cooperative relations, pages 213-237, 2000.

7. J. Golbeck. Computing and Applying Trust in Web-based Social Networks. PhD thesis, Department of Computer Science, University of Maryland, 2005.

8. J. Golbeck and J. A. Hendler. Inferring Binary Trust Relationships in Web-Based Social Networks. ACM Transactions on Internet Technology, 6(4):497-529, 2006.

9. C.-W. Hang, Y. Wang, and M. P. Singh. Operators for Propagating Trust and their Evaluation in Social Networks. In Proceedings of the 8th International Conference on Autonomous Agents and Multiagent Systems (AAMAS), pages 1025-1032, 2009.

10. A. Jøsang. A Logic for Uncertain Probabilities. International Journal of Uncertainty, Fuzziness and Knowledge-Based Systems, 9(3):279-311, 2001.

11. A. Jøsang and T. Bhuiyan. Optimal Trust Network Analysis with Subjective Logic. In Proceeding of the 2nd International Conference on Emerging Security Information, Systems and Technologies (SECURWARE), pages 179-184, 2008.

12. A. Jøsang, R. Hayward, and S. Pope. Trust Network Analysis with Subjective Logic. In Proceedings of the 29th Australasian Computer Science Conference (ACSC), pages 85-94, 2006.

13. A. Jøsang, R. Ismail, and C. Boyd. A Survey of Trust and Reputation Systems for Online Service Provision. Decision Support Systems, 43(2):618-644, 2007.

14. L. Li and Y. Wang. A Subjective Probability Based Deductive Approach to Global Trust Evaluation in Composite Services. In Proceedings of the 9th IEEE International Conference on Web Services (ICWS), pages 604-611, 2011.

15. G. Liu, Y. Wang, M. Orgun, and E. Lim. Finding the Optimal Social Trust Path for the Selection of Trustworthy Service Providers in Complex Social Networks. Services Computing, IEEE Transactions on, PP(99):1, 2011.

16. M. Richardson, R. Agrawal, and P. Domingos. Trust Management for the Semantic Web. In Proceedings of the 2nd International Semantic Web Conference (ISWC), pages 351-368, 2003.

17. E. M. Uslaner. The Moral Foundations of Trust. Cambridge, UK: Cambridge University Press, 2002.

18. P. Zhang, A. Durresi, and L. Barolli. Survey of trust management on various networks. In Proceedings of the 5th International Conference on Complex, Intelligent and Software Intensive Systems (CISIS), pages 219-226, 2011. 